\title{
Anomalous Cosmic Rays in the Heliosheath
}

\author{
A. C. Cummings a , E. C. Stone ${ }^{a}$, F. B. McDonald ${ }^{b}$, B. C. Heikkila ${ }^{c}$, N. \\ $\mathrm{Lal}^{\mathrm{c}}$, and W. R. Webber ${ }^{\mathrm{d}}$ \\ ${ }^{a}$ California Institute of Technology, Mail Code 220-47, Pasadena, CA 91125, USA \\ ${ }^{b}$ Institute for Physical Science and Technology, University of Maryland, College Park, MD 20742, USA \\ ${ }^{\circ} N A S A /$ Goddard Space Flight Center, Greenbelt, MD 20771, USA \\ dDepartment of Physics and Astronomy, New Mexico State University, Las Cruces, NM 88003, USA
}

\begin{abstract}
We report on Voyager 1 and 2 observations of anomalous cosmic rays in the outer heliosphere. The energy spectrum of anomalous cosmic ray helium as each spacecraft crossed the solar wind termination shock into the heliosheath remained modulated. Assuming the intensity gradient between the two spacecraft is purely radial, we find that radial gradients in the heliosheath of He with 11.6-22.3 MeV/nuc and with $\sim 61-73 \mathrm{MeV} /$ nuc are $4.9 \pm 1.2 \% / \mathrm{AU}$ and $0.0 \pm 0.5 \% / \mathrm{AU}$, respectively. Strong temporal variations of the $11.6-22.3 \mathrm{MeV} / \mathrm{nuc} \mathrm{He}$ intensity at both spacecraft were observed in 2005 just after Voyager 1 crossed the termination shock and while Voyager 2 was upstream. After 2006.0, the intensity variations are more moderate and likely due to a combination of spatial and temporal variations. As of early 2008 , the anomalous cosmic ray He energy spectrum has unfolded to what may be a source spectrum. The spectrum at Voyager 2 remains modulated. We examine three recent models of the origin of anomalous cosmic rays in light of these observations.
\end{abstract}

Keywords: Anomalous cosmic rays, solar wind termination shock, Voyager, shock acceleration PACS: $96.40 . \mathrm{Cd}, 96.40 . \mathrm{De}, 96.40 . \mathrm{Kk}, 96.50 . \mathrm{Pw}$

\section{INTRODUCTION}

Both Voyager spacecraft are now in the heliosheath. Voyager 1 (V1) crossed the solar wind termination shock (TS) on 16 December $2004^{1-4}$ and Voyager 2 (V2) crossed it several times on 30-31 August $2007^{5-9}$. Prior to Vl's crossing, the expectation was that as the spacecraft approached the TS, the anomalous cosmic ray (ACR) energy spectrum would unfold at low energies to its expected power-law shape from diffusive shock acceleration (see, e.g. ${ }^{10}$ ). These ACR particles were thought to be interstellar neutral gas that has entered the heliosphere, become ionized by the solar wind or by photons from the Sun ${ }^{11}$, and then carried out to the TS where they would be accelerated to high energies ${ }^{12}$. The unfolding of the low-energy ACR spectrum was not observed during the approach to and crossing of the TS by either spacecraft. Instead, the ACR He spectrum, for example, remained modulated and was relatively unperturbed as the spacecraft passed into the heliosheath. This was true for both V1 and V2 as shown in Fig. 1. We examine three models put forward to account for this observation.

\footnotetext{
CP1039, Particle Acceleration and Transport in the Heliosphere and Beyond $7^{\text {th }}$ Annual Astrophysics Conference edited by G. Li, Q. Hu, O. Verkhoglyadova, G. P. Zank, R. P. Lin, and J. Luhmann (O) 2008 American Institute of Physics 978-0-7354-0566-0/08/ $\$ 23.00$
} 


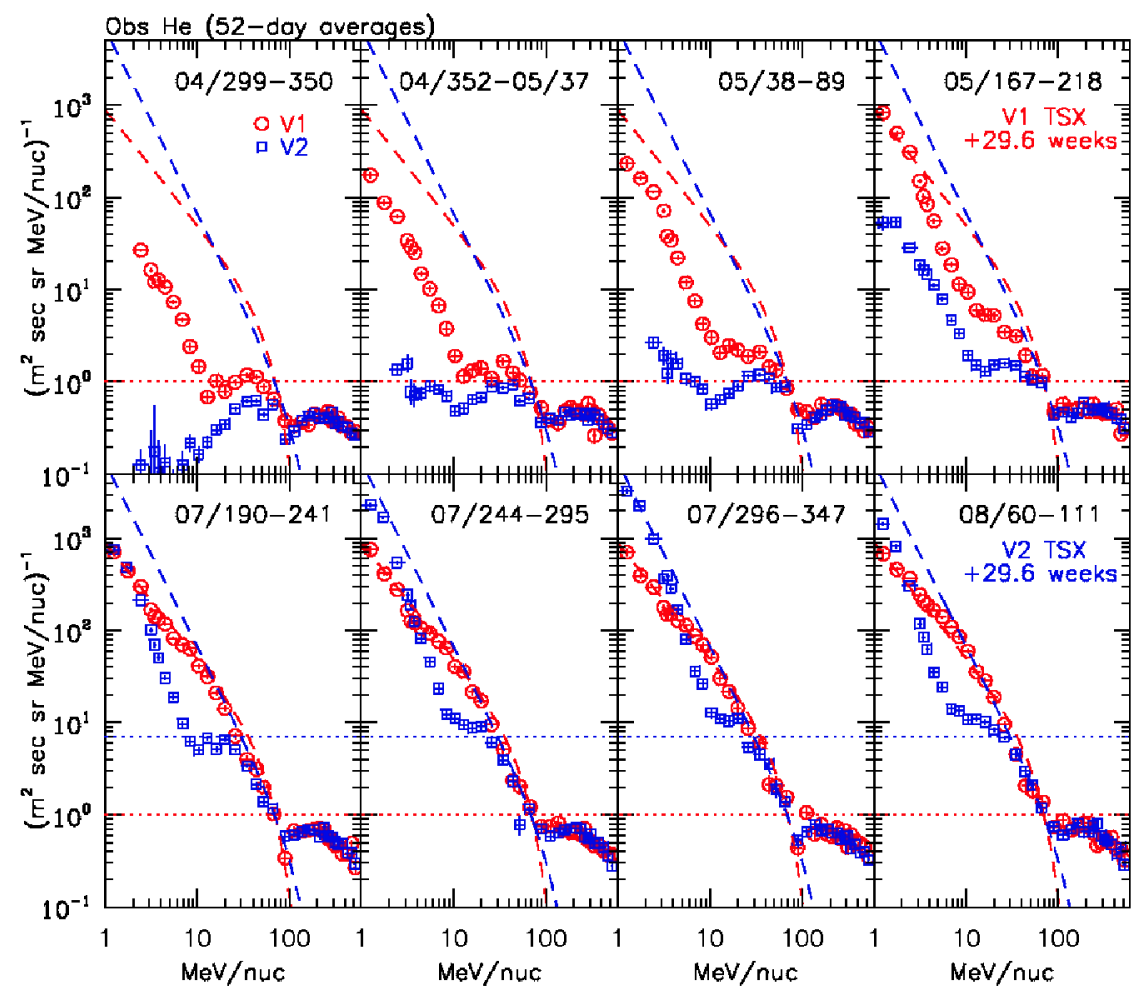

FIGURE 1. Fifty-two day averaged energy spectra of He at V1 and V2 for 8 time periods. V1 crossed the TS on day 351 of 2004, between the first two panels of the top row. The ACR portion of the energy spectrum is from $\sim 10-100 \mathrm{MeV} /$ nuc. Examples of possible source spectra at the TS are shown by the dashed lines. The ACR He spectrum remained in a modulated state during the shock crossings.

However, by 2008/60-111, the V1 energy spectrum may have unfolded to the ACR source spectrum.

\section{DYNAMICAL EFFECTS ON THE SHOCK}

Florinski and Zank ${ }^{13}$ showed that the interaction of a merged interaction region (MIR) with the TS could cause a decrease in the intensity of the shock at medium energies, resulting in energy spectra for $\mathrm{H}$ that resembled those in Fig. 1 for He. Jokipii $^{14}$ also suggested that motions and changes of the TS on time scales of the acceleration time of the particles would cause deviations from the expected shock accelerated spectra. While there was a factor of two decrease in the intensity of 11.6$22.3 \mathrm{MeV} /$ nuc He just before the V1 shock crossing, no similar decrease was apparent at the time of the V2 crossing. Thus, there is no evidence that transients caused the V2 intensity just after the shock to be only one-third the concurrent intensity observed at V1. 


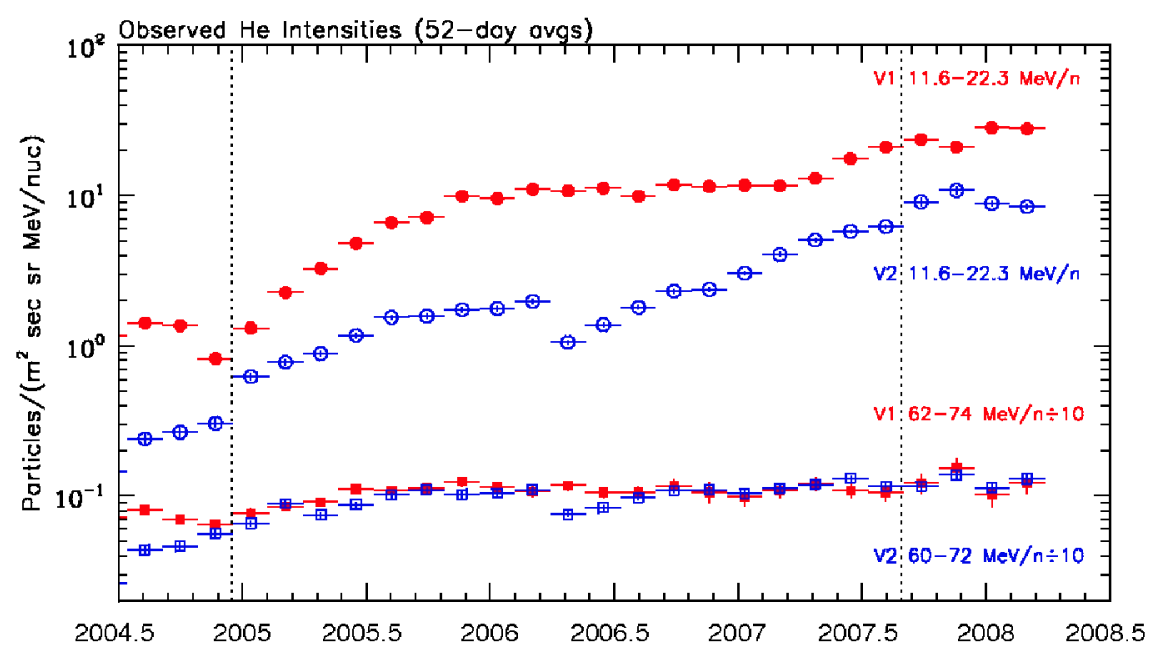

FIGURE 2. Intensities of mid- and high-energy $A C R$ He vs. time at V1 and V2. The two vertical dotted lines mark the times of the TS crossings by V1 and V2. Note the dip in the V1 intensity at 11.6$22.3 \mathrm{MeV} /$ nuc just prior to its TS crossing, an indication that an MIR or transient passed the spacecraft. The V2 profile shows no evidence of an MIR at the time of its TS crossing. In both cases, the energy spectrum of ACR He remained modulated as the TS was crossed (see Fig. 1).

\section{CONTINUOUS ACCELERATION IN HELIOSHEATH}

Several authors have proposed that some form of stochastic acceleration occurs in the heliosheath and that the observed energy spectrum of ACR He should continue to evolve towards its source shape as the observer moves further into the heliosheath ${ }^{15-17}$ As shown in Fig. 2, such evolution did take place in 2005 after V1 crossed the TS in December 2004. However, during 2005 a similar increase occurred at V2 which was upstream of the shock, indicating that the change was mainly temporal rather than due to a large gradient in the heliosheath. An estimate of the radial gradient in the heliosheath can be derived from the intensities at V1 and V2 during the four periods in Fig. 2 after V2 crossed the TS in late August 2007. At 11.6-22.3 MeV/nuc, the average gradient is $4.9 \pm 1.2 \% / \mathrm{AU}$, where the uncertainty is the sample standard deviation of the four gradient determinations. Such a gradient could perhaps account for the change in V1 intensity from 2006.0 to early 2007, but not for the much larger change that occurred before that time. So, it is likely that most of the factor of $\sim 10$ increase in intensity between 2005.0 and 2006.0 was due to a decreasing level of modulation and not due to a gradient in the heliosheath. This is also consistent with the absence of such a large increase in intensity following the V2 crossing. The gradient at $\sim 61-73 \mathrm{MeV} / \mathrm{nuc}$ is $0.0 \pm 0.5 \% / \mathrm{AU}$, suggesting that at these energies the heliosheath is filled uniformly and that the spacecraft are likely observing the source intensity.

In Fig. 3 we show the expected radial intensity variation of the intensity of 20 $\mathrm{MeV} / \mathrm{nuc} \mathrm{He}$ at $\mathrm{Vl}$ from Ferreira et al. ${ }^{15}$ based on a combination of diffusive shock 
acceleration, adiabatic heating, and stochastic acceleration, with the latter process being the dominant one. Observations of 19-25 MeV/nuc He are shown for comparison. While the calculated curve resembles the observations, the fast initial rise after the TS crossing at 94 AU is likely a temporal effect rather than due to a spatial gradient as discussed above. From 97 to $106 \mathrm{AU}$, however, the calculated intensity increases at $\sim 11 \%$ /AU, similar to the overall rate of increase of the observations. However, as noted above, the instantaneous radial gradient measured just after the V2 TS crossing was about a factor of two lower than this value. In addition, the large intensity increase observed by V1 immediately after the shock was not observed at V2, indicating that the V1 increase was likely dominated by a temporal change (Fig. 3 ). This model deserves further consideration, particularly to incorporate the temporal component of the intensity in a self-consistent way.
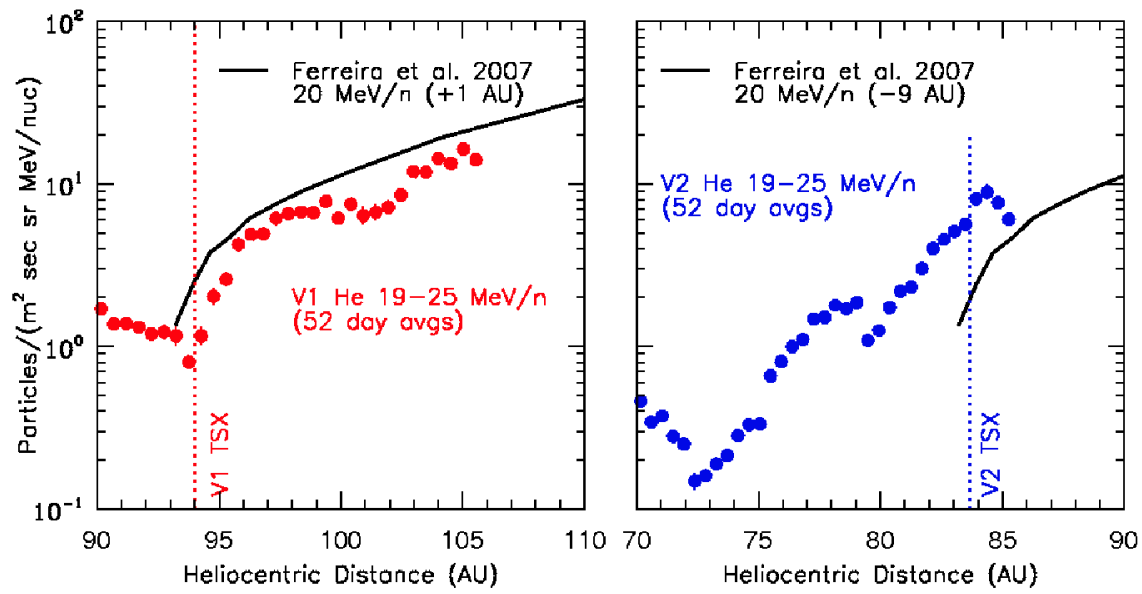

FIGURE 3. (left) Calculated intensity profile of $20 \mathrm{MeV} /$ nuc He along the V1 trajectory from Ferreira et al. ${ }^{15}$ The curve has been shifted by +1 AU to account for the actual TS crossing at $94 \mathrm{AU}$, marked with the vertical dotted line, rather than the value of $93 \mathrm{AU}$ used by Ferreira et al. Solid points are 52-day averages of V1 He with 19-25 MeV/nuc. (right) Same as left except for V2 and solid curve from Ferreira et al. ${ }^{15}$ has been shifted -9 AU.

\section{ACCELERATION AT SITE REMOTE FROM NOSE REGION OF TS}

Another possibility is that the blunt noise of the heliosphere is not a region conducive to acceleration of ACRs to high energies if there is little cross-field diffusion ${ }^{18-21}$. Because the interplanetary magnetic field lines convect through the shock rather rapidly and do not stay in contact with the shock in the nose region, the particles do not have time to be accelerated to high energies. Rather, in this scenario the high energy ACRs would be generated back along the flanks or tail of the heliosphere where the connection times are much longer. Both Voyager spacecraft penetrated into the heliosheath in the nose region, with V1 crossing within 2 degrees 
in longitude from the nose (defined as the direction of the inflowing interstellar neutral He at $\lambda, \beta=254.7^{\circ}, 5.2^{\circ}$ in solar ecliptic coordinates ${ }^{22}$ ) at $34.7^{\circ} \mathrm{N}$ latitude and $253^{\circ}$ longitude, and with $\mathrm{V} 2$ crossing $34.1^{\circ}$ in longitude from the nose at $31.6^{\circ} \mathrm{S}$ latitude and $288.8^{\circ}$ longitude. The TS in the nose region would be expected to accelerate low energy ACRs, which would then be convected to the spacecraft in the heliosheath. This low energy component is visible in Fig. 1 at energies below $\sim 10 \mathrm{MeV} /$ nuc. Indeed, after the V2 TS crossing, the low energy He intensity below $2-3 \mathrm{MeV} /$ nuc is higher at V2 than at V1, suggesting differences in the local acceleration conditions at different locations along the TS.

This model would also predict a positive radial gradient of mid-energy ACRs in the heliosheath as they would arrive at the spacecraft after propagating in longitude from the tail or flank regions to the nose region along the magnetic field lines. At higher energies, where the diffusive mean free pathlengths are large, the intensity would be expected to be approximately uniform in the heliosheath, in agreement with the near zero gradient observed for He between V1 and V2 at $61-73 \mathrm{MeV} /$ nuc (see Fig. 2).

A significant longitudinal dependence of the energy spectrum would be expected in this model. In Fig. 4 we show model calculations of the energy spectrum of ACR $\mathrm{H}$ at four longitudes measured from the nose of the heliosphere from Schwadron et al. ${ }^{23}$.

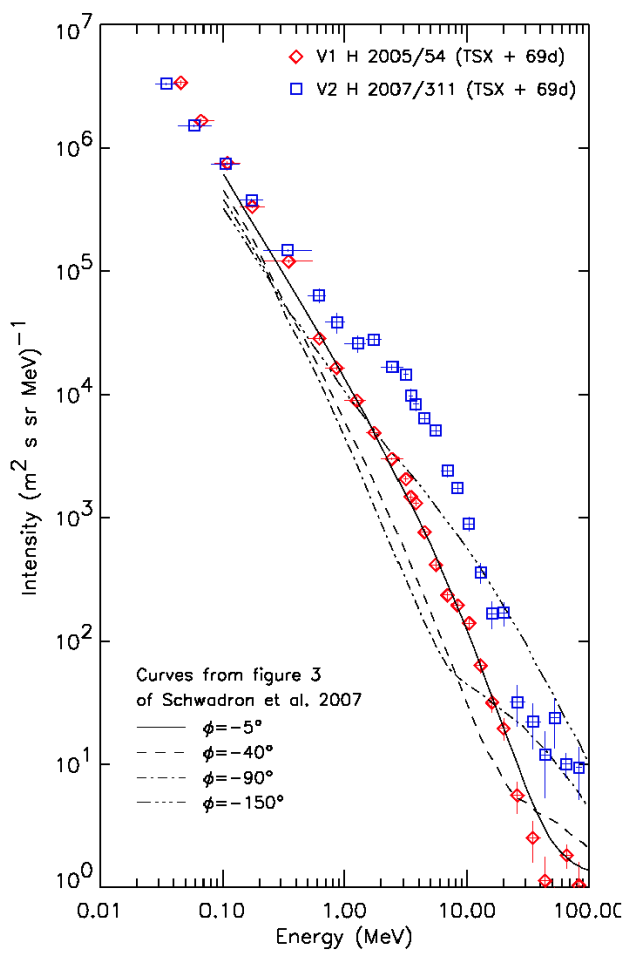

FIGURE 4. Calculated energy spectra of ACR H from at four longitudes away from the nose of the $\mathrm{TS}^{23}$. The symbols show observations at V1 (open diamonds) and at V2 (open squares). Both spectra are one-day averages 69 days after the respective shock crossings. 
The V1 data compare well to the solid line for $-5^{\circ}$ from the nose, the approximate position of V1 when it crossed the TS. However, the V2 observations do not agree with the dashed line at $-40^{\circ}$ from the nose, its approximate TS crossing location. Part of the reason is the strong temporal intensity variation that took place at both spacecraft between the times of the two shock crossings.

\section{DISCUSSION AND CONCLUSIONS}

Of the three models discussed here, the two that feature a prediction of a positive radial gradient in the heliosheath show the most promise. There is no evidence that transients were responsible for the observation that the V2 intensity at the shock was lower than at V1 in the heliosheath. However, the other two models must consider temporal changes as well as spatial gradients.

\section{ACKNOWLEDGMENTS}

We appreciate the availability of the $\mathrm{LECP}^{24}$ data used in Fig. 4. This work was supported by NASA under contract NAS7-03001.

\section{REFERENCES}

L. F. Burlaga, N. F. Ness, M. H. Acuna et al., Science 309, 2027 (2005).

R. B. Decker, S. M. Krimigis, E. C. Roelof et al., Science 309, 2020 (2005).

D. A. Gurnett and W. S. Kurth, Science 309, 2025 (2005).

E. C. Stone, A. C. Cummings, F. B. McDonald et al., Science 309, 2017 (2005).

L. F. Burlaga, N. F. Ness, M. H. Acuna et al., Nature (submitted) (2008).

R. B. Decker, S. M. Krimigis, E. C. Roelof et al., Nature (submitted) (2008).

D. A. Gurnett and W. S. Kurth, Nature (submitted) (2008).

John D. Richardson, Justin C. Kasper, C. Wang et al., Nature (submitted) (2008).

E. C. Stone, A. C. Cummings, F. B. McDonald et al., Nature (submitted) (2008).

R. D. Blandford and J. P. Ostriker, Astrophys. J. Lett. 221, L29 (1978).

L. A. Fisk, B. Kozlovsky, and R. Ramaty, Astrophys. J. Lett. 190, L35 (1974).

M. E. Pesses, J. R. Jokipii, and D. Eichler, Astrophys. J. Lett. 246, L85 (1981).

V. Florinski and G. P. Zank, Geophysical Research Letters 33, L15110 (2006).

J. R. Jokipii, in American Institute of Physics Conference Series (2006), Vol. 858, pp. 143.

S. E. S. Ferreira, M. S. Potgieter, and K. Scherer, Journal of Geophysical Research (Space Physics) 112 , 11101 (2007).

L. A. Fisk, G. Gloeckler, and T. H. Zurbuchen, Astrophysical Journal 644, 631 (2006).

H. Moraal, R. A. Caballero-Lopez, and V. Ptuskin, presented at the 30th International Cosmic Ray Conference, Merida, Mexico, 2008 (unpublished).

J. Kóta, presented at the 30th International Cosmic Ray Conference, Merida, Mexico, 2008 (unpublished).

9 D. J. McComas and N. A. Schwadron, Geophysical Research Letters 33, L04102 (2006).

N. A. Schwadron and D. J. MoComas, in American Institute of Physics Conference Series (2006), Vol. 858 , pp. 165.

N. A. Schwadron, M. A. Lee, and D. J. MeComas, Astrophysical Journal 675, 1584 (2008).

R. Lallement, E. Quémerais, J. L. Bertaux et al., Science 307, 1447 (2005).

N. A. Schwadron, M. A. Lee, and D. J. McComas, Astrophys. J. (in press) (2008).

S. M. Krimigis, T. P. Armstrong, W. I. Axford et al., Space Sci. Rev. 21, 329 (1977).
} 